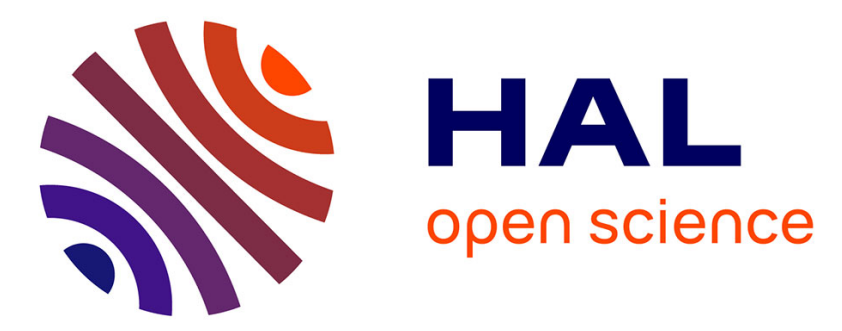

\title{
Expected Equivalent Magnetic Noise Spectral Density of Magnetoelectric Composites as Magnetic sensors: From Theory to Experiments
}

\author{
Xin Zhuang, Sébastien Saez, Marc Lam Chok Sing, Christophe Cordier, \\ Christophe Dolabdjian, Jiefang Li, K. Mclaughlin, Dwight Viehland
}

\section{To cite this version:}

Xin Zhuang, Sébastien Saez, Marc Lam Chok Sing, Christophe Cordier, Christophe Dolabdjian, et al.. Expected Equivalent Magnetic Noise Spectral Density of Magnetoelectric Composites as Magnetic sensors: From Theory to Experiments. MRS Proceedings, 2012, 1398, 12 p. 10.1557/opl.2012.767. hal-01062248

\section{HAL Id: hal-01062248 \\ https://hal.science/hal-01062248}

Submitted on 9 Sep 2014

HAL is a multi-disciplinary open access archive for the deposit and dissemination of scientific research documents, whether they are published or not. The documents may come from teaching and research institutions in France or abroad, or from public or private research centers.
L'archive ouverte pluridisciplinaire HAL, est destinée au dépôt et à la diffusion de documents scientifiques de niveau recherche, publiés ou non, émanant des établissements d'enseignement et de recherche français ou étrangers, des laboratoires publics ou privés. 


\title{
Expected Equivalent Magnetic Noise Spectral Density of Magnetoelectric Composites as Magnetic sensors: From Theory to Experiments
}

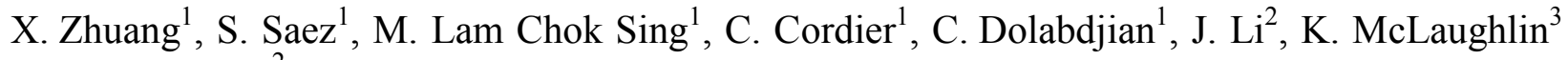 \\ and D. Viehland ${ }^{2}$ \\ ${ }^{1}$ Groupe de Recherche en Informatique, Image, Automatique et Instrumentation de Caen \\ (GREYC), CNRS UMR 6072 - ENSICAEN and the University of Caen Basse Normandie, \\ France 14050 Caen Cedex, \\ ${ }^{2}$ Materials Science and Engineering, Virginia Tech, Virginia 24061, USA \\ ${ }^{3}$ SAIC, McLean, Virginia, USA
}

\begin{abstract}
With the development of applications involving high sensitivity ferromagneticferroelectric laminates, a systematic analysis of the noise floor for magneto-electric (ME) laminated sensor has become crucial. We report and discuss the results of such an analysis on the noise floor of magnetostrictive-piezoelectric laminates in terms of the magnetic noise spectral density at room temperature. The noise floor of highly sensitive ME laminates with a JFET charge amplifier detection method has been studied. A good correlation was found between the theoretical and experimental noise curves within the measurement bandwidth. The dominating noise sources were found to include the dielectric loss noise, mechanical loss noise of the magneto-electric laminates and the noise sources of the charge amplifier. By using an appropriate low noise JFET charge amplifier, the noise contributions from the amplifier can be made negligible, enabling the measurement of the intrinsic noise of the ME laminates sensor. Thus, we have shown that at low frequencies, below the resonant frequency, the dielectric loss noise predominates with a one-per-root-frequency dependence whereas, around the resonance, the mechanical loss noise prevails over all other noise sources as expected from our theoretical analysis.
\end{abstract}

\section{INTRODUCTION}

Magneto-electric (ME) laminate sensors with integrated electronics have been used to measure small magnetic signals due to their high magnetostrictive and piezoelectric effects. ${ }^{1-5} \mathrm{~A}$ low noise ME magnetometer is composed of ME laminates and a low noise charge or voltage amplifier used for amplifying the signals generated by the ME laminates. ${ }^{6,7,8}$ Advantages of such a system include high sensitivity, low noise level and a wide dynamic range.

Significant progress has been achieved in the development of high sensitivity ME laminates by using highly magnetostrictive and piezoelectric materials. ${ }^{9-12}$ In order to enhance the magnetic signal sensitivity, several structures of ME laminates have been fabricated and studied. Among these designs, one type of structure involving interdigital electrodes and permanent magnets showed excellent performance under free boundaries conditions. The interdigital electrodes ${ }^{13}$ attached on the piezoelectric materials collect the electric signals generated by the ME laminates along their electric polarization direction. A biasing magnetic field, produced by magnets, was used in order to enhance the sensor sensitivity to time varying magnetic signals by a factor ranging from several dozens to several hundreds. ${ }^{9}$ 
Due to high performance magnetostrictive and piezoelectric materials, ME laminates can achieve a high magnetic signal sensitivity. With regards to applications involving these materials, intrinsic noise sources often limit their sensing performance. ${ }^{14}$ Intrinsic noise in the ME laminates can have several origins. Random rotations and displacements of domains within the piezoelectric layers result in high dielectric loss noise at low frequencies, ${ }^{15}$ migration of oxygen vacancies in piezoelectric materials leads to a resistance degradation producing an equivalent thermal noise at quasi-static frequencies ${ }^{16}$ and miniature or small laminates are susceptible to mechanical noise resulting from molecular agitations. ${ }^{14,17}$

Electric signals generated by ME laminates are amplified by either a charge or a voltage amplifier electronics. Hence, noise sources of the electronics pose another limitation to detection systems. $^{7,18,19}$ Usually, the equivalent input current and voltage noise for a given amplifier dominates the electronic noise floor at low and high frequencies, respectively. Therefore, noise contributions from a perfectly designed amplifier must be lower than the noise floor of $\mathrm{ME}$ laminates in the working bandwidth. In addition, concerns about environmental perturbations must be taken into consideration during measurements. Vibrations and acoustic waves may result in an excess noise level, higher than that predicted by the noise floor models at low frequencies, for example below $1 \mathrm{~Hz}$.

In this paper, we present a noise analysis and give the expression of the equivalent magnetic noise floor for the ME laminates with its associated amplifier. Moreover, experimental noise curves have been compared to those given by our theoretical analysis.

\section{NOISE ANALYSIS OF ME LAMINATES ASSOCIATED WITH A CHARGE AMPLIFIER}

The magnetic signal sensitivity of the ME laminates associated with a charge amplifier is defined by the charge coefficient $\alpha_{M E}^{Q}$ in $\mathrm{C} / \mathrm{T}$ units. Besides, the transfer function for such a system is given by

$$
T_{r}=\frac{\alpha_{M E}^{Q}}{C_{1}}
$$

where the feedback capacitor $C_{1}$ of the charge amplifier determines the magnetic field signal gain for a given ME laminates sensor. However, a too small feedback capacitor tends to push the low cutoff frequency to much higher values and this can lead to a bad frequency response. A large resistance $R_{1}$ is required to get rid of this influence and to stabilize the system. Moreover, thermal noise from such a large bias resistance results in a lower noise contribution, in terms of applied magnetic signals.

All the intrinsic noise sources referred to the input terminal of ME laminates define the equivalent magnetic noise ${ }^{19}$. This noise spectral density characterizes the performance of magnetic sensors and magnetometers. In order to find the overall expression of the equivalent magnetic noise floor, the electric loss noise $i_{n_{-} \text {piezo }}$ and the mechanical noise $a_{n}$ of the ME laminates, the bias resistance thermal noise $i_{n_{-} R_{I}}$ as well as the input current noise $i_{n_{-} a m p}$ and voltage noise $e_{n_{-} \text {amp }}$ of the charge amplifier were taken into account as the main intrinsic noise sources in the following analysis. Noise contributions from the resistance degradation of the piezoelectric material were disregarded because of the low frequency bandwidth. In addition, environmental vibration perturbations were also disregarded, supposing a sufficiently quiet 
measurement environment. In our experiments, we a second amplifier was used to further amplify the voltage at the output of the charge amplifier. The voltage gain of this additional amplifier will be noted hereafter as "Gain". 


\section{A) INTRINSIC NOISE OF ME LAMINATES}

The intrinsic noise of the ME laminates determines the fundamental noise limit. The piezoelectric loss noise related to the dielectric relaxation and the mechanical noise caused by the molecular agitations are taken into account as the two main noise sources for the ME laminates. The first noise source determines low frequency noise floor, whereas the mechanical noise sometimes determines high frequency noise floor because of its white noise contribution in that frequency range. The piezoelectric loss noise can be written as

$$
i_{n_{-} \text {piezo }}(f)=\sqrt{4 k_{B} T 2 \pi f C \tan \left(\delta_{\text {piezo }}\right)}
$$

where $k_{B}$ is the Boltzmann constant, $T$ the temperature in Kelvin, $\omega$ the angular frequency, $C$ the laminates capacitance and $\tan \left(\delta_{\text {piezo }}\right)$ the piezoelectric loss factor which is dependent on the frequency. From a previous analysis, ${ }^{19}$ we know that the equivalent magnetic noise contribution referring to the piezoelectric loss noise can be written as

$$
b_{n_{-} \text {piezo }}(f)=\left(\mu_{0} \frac{n s_{3, p}^{D}+(1-n) s_{33, m}^{H}\left(1-k_{3, p}^{2}\right)}{n d_{33, m} g_{33, p}}\right) \sqrt{\frac{4 k_{B} T}{2 \pi f \varepsilon_{33}^{S} m l w t_{p}} \tan \left(\delta_{\text {piezo }}\right)}
$$

where $b_{n_{-} \text {piezo }}(f)$ represents the equivalent magnetic noise spectral density. $\mu_{0}$ is the magnetic permeability. $n$ is the magnetostrictive layers thickness ratio. $s_{33, p}^{D}\left(=s_{33, p}^{E}\left(1-k_{33, p}^{2}\right)\right)$ and $s_{33, m}^{H}$ are the flexibilities for the magnetostrictive and piezoelectric materials. $k_{33, p}$ and $\varepsilon_{33}^{S}$ are the coupling constant and the dielectric constant of the piezoelectric material. $d_{33, m}$ and $g_{33, p}$ are the magnetostrictive and piezoelectric constants. $l, w$ and $t_{p}$ are respectively the length, width and thickness of the laminates. $f$ is the frequency of the sensed magnetic signal.

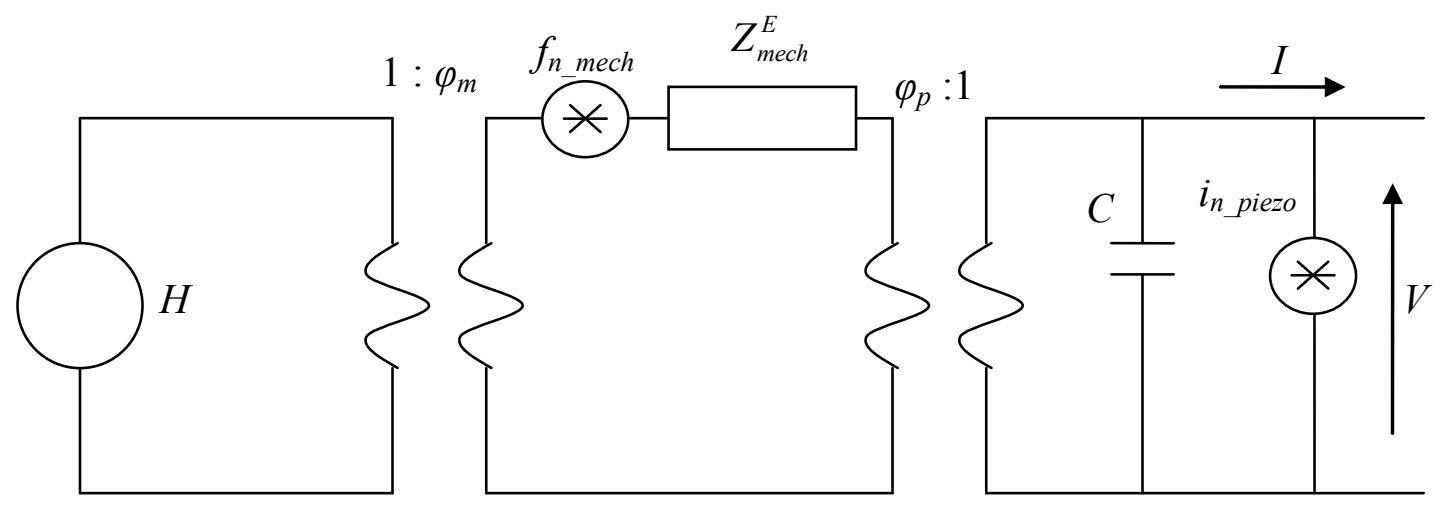

Figure 1: Simplified equivalent circuit under free boundary conditions at low frequency.

Since the classical mechanical acceleration noise formula is given by ${ }^{14,17}$, $a_{n}=\sqrt{\frac{4 k_{B} T \omega_{\text {res }}}{m_{\text {mass }} Q_{\text {mech }}}}$, expressed in $\mathrm{g} / \sqrt{ } \mathrm{Hz}$ units, the mechanical force noise can be written as $f_{n_{-} \text {mech }}=\sqrt{4 k_{B} T \omega_{\text {res }} m_{\text {mass }} \tan \left(\delta_{\text {mech }}\right)}$, where $\omega_{\text {res }}$ is the resonant angular frequency, $m_{\text {mass }}$ and $Q_{m e c h}$ are the mass and mechanical quality factor of the laminates with $m_{\text {mass }}\left(=m \times m_{\text {mass_seg }}\right)$ and 
$Q_{\text {mech }}\left(=\frac{1}{\tan \left(\delta_{\text {mech }}\right)}\right) \cdot m_{\text {mass_seg }}$ is the mass of a single piezoelectric segment between two adjacent interdigital electrodes, $m$ the number of segments and $\tan \left(\delta_{\text {mech }}\right)$ the sensor mechanical loss. This noise source $f_{n \_m e c h}$ is represented by the sensor model shown in figure 1 . $Z_{\text {mech }}^{E}=-j\left(\frac{n}{s_{33, m}^{H}}+\frac{1-n}{s_{33, p}^{E}}\right) \frac{A}{k v l}$ defines the mechanical impedance, with $A\left(=t_{\text {lam }} \times w\right)$ the crosssectional area of the laminates, $k$ the elastic wave number and $v$ the elastic wave speed. $\varphi_{p}=\frac{A_{p}}{l} \frac{d_{33, p}}{s_{33, p}^{E}}$ and $\varphi_{m}=\frac{A_{m}}{l} \frac{d_{33, m}}{s_{33, m}^{H}}$ are respectively elasto-electric and magneto-elastic coupling coefficients, with $A_{p}\left(=t_{p} \times w\right)$ the piezoelectric layer cross-sectional area $A_{m}\left(=t_{m} \times w\right)$ the magnetostrictive layer cross-sectional area. $C$ is the laminates capacitance for the $m$ L-L pushpull mode, which corresponds to the mode of the tested laminates with $C=m C_{0}=m \varepsilon_{33}^{S} \frac{A_{p}}{l_{\text {seg }}}$ where $C_{0}$ and $l_{\text {seg }}$ are the capacitance and length of each piezoelectric segment between two interdigital electrodes. From figure 1, we can find the expression of $q_{n_{-} \text {mech }}$ the charge noise resulting from mechanical noise as

$$
q_{n_{-} \text {mech }}=\frac{d_{33, p}}{1-\left(2-\frac{s_{33, p}^{E}}{s_{33, m}^{H}}\right) n+\left(1-\frac{s_{33, p}^{E}}{s_{33, m}^{H}}\right) n^{2}} \sqrt{4 k_{B} T \omega_{\text {res }} m_{\text {mass }} \tan \left(\delta_{\text {mech }}\right)}
$$

Referring to our measurement scheme where we used a low noise JFET charge amplifier followed by a second stage amplifier having a voltage gain noted as "Gain", we can write the final output voltage noise density $e_{n_{-} \text {mech }}($ in $\mathrm{V} / \sqrt{ } \mathrm{Hz}$ ) as

$$
e_{n_{-} \text {mech }}=\frac{q_{n_{-} \text {mech }}}{C_{1}} \times \text { Gain }=\frac{\text { Gain }}{C_{1}} \frac{d_{33, p}}{1-\left(2-\frac{s_{33, p}^{E}}{s_{33, m}^{H}}\right) n+\left(1-\frac{s_{33, p}^{E}}{s_{33, m}^{H}}\right) n^{2}} \sqrt{4 k_{B} T \omega_{\text {res }} m_{\text {mass }} \tan \left(\delta_{\text {mech }}\right)}
$$

where $C_{l}(=10 \mathrm{pF})$ is the feedback capacitor and a Gain value of 50. The piezoelectric constant $d_{33, p}$ determines the output voltage noise floor. The output voltage noise spectral density $e_{n_{-} m e c h}$ res around the laminates resonance can be written as ${ }^{19,20}$,

$$
e_{n_{-} \text {mech_res }}=\frac{8 Q_{\text {mech }}}{\pi^{2}} e_{n_{-} \text {mech }}
$$

So, the equivalent magnetic noise corresponding to the mechanical noise source can be represented as

$$
b_{n_{-} \text {mech }}=\frac{\text { Gain }}{T_{r} \times C_{1}} \frac{d_{33, p}}{\left(1-\frac{s_{33, p}^{E}}{s_{33, m}^{H}}\right) n^{2}-\left(2-\frac{s_{33, p}^{E}}{s_{33, m}^{H}}\right) n+1} \sqrt{4 k_{B} T \omega_{\text {res }} m_{\text {mass }} \tan \left(\delta_{\text {mech }}\right)}
$$


Taking into account equation (1) and the charge coefficient $\alpha_{M E}^{Q}$ for the L-L push pull mode with $\mathrm{m}$ piezoelectric segments, which we note as $\mathrm{m}$ L-L mode, we have $\alpha_{M E}^{Q}=\frac{m}{\mu_{0}} \frac{n t_{p} w d_{33, m} d_{33, p}}{n s_{33}^{E}+(1-n) s_{33}^{H}}$ and the equivalent magnetic noise can be obtained as

$$
b_{n_{-} \text {mech }}=\frac{\mu_{0} s_{33, m}^{H}}{m w t_{p} n(1-n) d_{33, m}} \sqrt{4 k_{B} T \omega_{\text {res }} m_{\text {mass }} \tan \left(\delta_{\text {mech }}\right)}
$$

Reminding the longitudinal resonant frequency $\omega_{\text {res }}=\frac{\pi}{l} \sqrt{\frac{n s_{33, p}^{E}+(1-n) s_{33, m}^{H}}{s_{33, p}^{E} s_{33, m}^{H}\left((1-n) \rho_{p}+n \rho_{m}\right)}}, \quad$ the equivalent magnetic noise $b_{n \_ \text {mech }}$ is represented as

$$
b_{n_{-} \text {mech }}=\frac{\mu_{0} s_{33, m}^{H}}{m n(1-n) d_{33, m}} \sqrt{\frac{4 k_{B} T \pi \tan \left(\delta_{\text {mech }}\right) \sqrt{\frac{\left(\left(n s_{33, p}^{E}+(1-n) s_{33, m}^{H}\right)\right)\left((1-n) \rho_{p}+n \rho_{m}\right)}{s_{33, p}^{E} s_{33, m}^{H}}}}{w t_{p}(1-n)}}
$$

Equation (9) shows that the equivalent magnetic noise $b_{n \text { mech }}$ is frequency independent. We can deduce from equation (8) that ME laminates with a high resonant frequency, a big mass or a low mechanical quality factor will lead to a higher equivalent magnetic noise floor. Meanwhile, we notice that the equivalent magnetic noise floor is independent of the piezoelectric material coefficients.

\section{B) INTRINSIC NOISE OF THE CHARGE AMPLIFIER}

One of our goals was to find out the performance of ME laminates associated with a charge amplifier. Therefore, we searched for an expression of the intrinsic equivalent magnetic noise floor referred to the input terminal of the ME laminates by using the noise model of the circuit given in figure 2 .

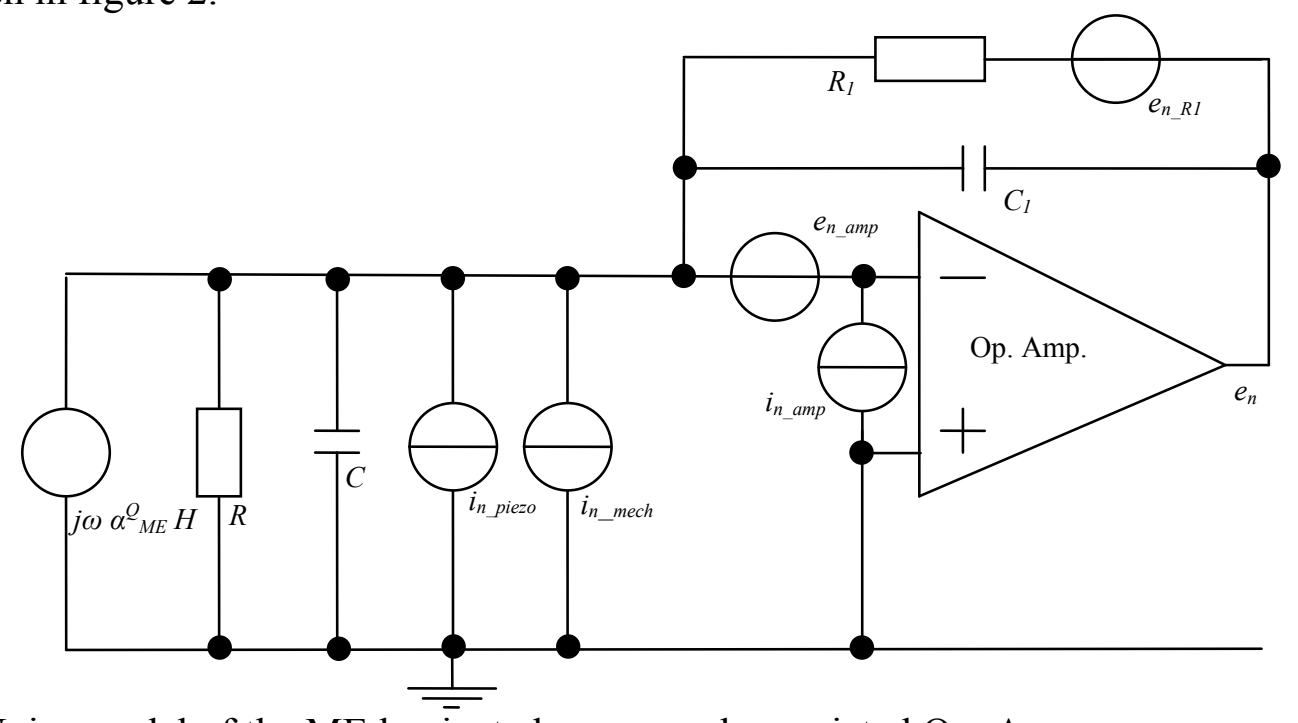

Figure 2: Noise model of the ME laminated sensor and associated Op. Amp. 
According to the noise analysis of the charge amplifier connected to the ME laminates sensor, the output voltage noise spectral density $e_{n_{-} \text {elec }}$ at the Op. Amp. in figure 2 can be written as

$$
e_{n_{-} \text {elec }}(f)=\left\{\left(\frac{R_{1}}{\sqrt{\left(2 \pi f R_{1} C_{1}\right)^{2}+1}} \sqrt{\frac{4 k_{B} T}{R_{1}}}\right)^{2}+\left(\left(\frac{R+R_{1}}{R_{i}}\right) \frac{\sqrt{\left(2 \pi f R_{p} C_{p}\right)^{2}+1}}{\sqrt{\left(2 \pi f R_{1} C_{1}\right)^{2}+1}} e_{n_{-} \text {amp }}\right)^{2}+\left(\frac{R_{1}}{\sqrt{\left(2 \pi f R_{1} C_{1}\right)^{2}+1}} i_{n_{-} \text {amp }}\right)^{2}\right\}^{1 / 2}
$$

where $R$ is the equivalent resistance of the piezoelectric laminates. $R_{p}$ is the value of $R$ in parallel with $R_{l}, C_{p}$ is equal to $C$ and $C_{l}$ in parallel. Despite the fact that the piezoelectric material is not a perfect insulator, we suppose that $R$ is large enough so that its noise contribution can be neglected.

The thermal noise of the bias resistance $R_{l}$ and the current noise of the amplifier contributions are respectively the first and third terms of the right hand side of equation (10). Both of them present a noise level decreasing with frequency, having a $1 / \mathrm{f}$ dependency. The equivalent voltage noise of the amplifier correspond to second term of equation (10) and it constitutes the white noise floor. Thus, the input magnetic noise spectral density for the charge amplifier can be written as

$$
b_{n_{-} \text {elec }}(f)=\frac{1}{\alpha_{M E}^{Q}} \sqrt{\frac{1}{(2 \pi f)^{2}}\left(i_{n_{-} a m p}^{2}(f)+\frac{4 k_{B} T}{R_{1}}\right)+\left(C_{1}+C\right)^{2} e_{n_{-a m p}}^{2}(f)}
$$

From equation (11), we can see that the equivalent magnetic noise floor resulting from the noise sources of a JFET charge amplifier is, generally, determined by the charge coefficient of the ME laminates, the current noise and voltage noise from the amplifier.

\section{C) OVERALL NOISE FLOOR CONSIDERING LAMINATES AND ELECTRONICS NOISE SOURCES}

Taking into account all the noise sources from the laminates as well as from the charge amplifier, we can write the complete expression of overall noise power spectrum floor

$$
\begin{aligned}
e_{n}^{2}(f) & =\operatorname{Gain} \times\left\{\left(\frac{2 \pi f R_{1}}{\sqrt{\left(2 \pi f R_{1} C_{1}\right)^{2}+1}} \frac{d_{33, p}}{\left(1-\frac{s_{33, p}^{E}}{s_{33, m}^{H}}\right) n^{2}-\left(2-\frac{s_{33, p}^{E}}{s_{33, m}^{H}}\right) n+1} \sqrt{4 k_{B} T \omega_{\text {res }} m_{\text {mass }} \tan \left(\delta_{\text {mech }}\right)}\right)^{2}\right. \\
& +\left(\frac{R_{1}}{\sqrt{\left(2 \pi f R_{1} C_{1}\right)^{2}+1}} \sqrt{4 k_{B} T C 2 \pi f \tan \left(\delta_{\text {piezo }}\right)}\right)^{2}+\left(\frac{R_{1}}{\sqrt{\left(2 \pi f R_{1} C_{1}\right)^{2}+1}} \sqrt{\frac{4 k_{B} T}{R_{1}}}\right)^{2} \\
& +\left(\left(\frac{R+R_{1}}{R}\right) \frac{\sqrt{\left(2 \pi f R_{p} C_{p}\right)^{2}+1}}{\sqrt{\left(2 \pi f R_{1} C_{1}\right)^{2}+1}} e_{n_{-} \text {amp }} \sqrt{1+\frac{f_{e_{n} \text { anp }}}{f}}\right)^{2} \\
& \left.+\left(\frac{R_{1}}{\sqrt{\left(2 \pi f R_{1} C_{1}\right)^{2}+1}} i_{n_{-} \text {amp }} \sqrt{1+\frac{f}{f_{i_{-a \text { anp }}}}}\right)^{2}\right\}
\end{aligned}
$$


The equivalent magnetic noise floor $b_{n}(f)$ can then be obtained with the help of equations (3), (8) and (11), as

$$
b_{n}(f)=\frac{1}{\alpha_{M E}^{Q}} \sqrt{\frac{1}{(2 \pi f)^{2}}\left(i_{n_{-} \text {loss }}^{2}(f)+i_{n_{-} \text {amp }}^{2}(f)+\frac{4 k_{B} T}{R_{1}}\right)+q_{n_{-} \text {mech }}^{2}+\left(C_{1}+C\right)^{2} e_{n_{-} \text {amp }}^{2}(f)} .
$$

Considering the expressions of the intrinsic noise sources from both the charge amplifier and the ME laminates, the equivalent magnetic noise power spectrum is given by

$$
\begin{gathered}
b_{n}^{2}(f)=\frac{1}{m^{2}}\left(\mu_{0} \frac{n s_{33, p}^{E}+(1-n) s_{33, m}^{H}}{n t_{p} w d_{33, m} d_{33, p}}\right)^{2}\left[\frac{1}{(2 \pi f)^{2}}\left(i_{n_{-} \text {amp }}^{2}(f)+\frac{4 k_{B} T}{R_{1}}\right)\right] \\
+\frac{1}{m}\left(\mu_{0} \frac{n s_{33, p}^{E}+(1-n) s_{33, m}^{H}}{n d_{33, m} d_{33, p}}\right)^{2} \frac{4 k_{B} T}{2 \pi f \varepsilon_{33}^{S} l_{\text {seg }} w t_{p}} \tan \left(\delta_{\text {piezo }}\right) \\
+\frac{1}{m}\left(\mu_{0} \frac{s_{33, m}^{H}}{t_{p} w n(1-n) d_{33, m}}\right)^{2} 4 k_{B} T \omega_{\text {res }_{\text {mass }} \text { seg }} m_{\text {mech }} \tan \left(\delta_{\text {mech }}\right) \\
+\frac{1}{m^{2}}\left(\mu_{0} \frac{n s_{33, p}^{E}+(1-n) s_{33, m}^{H}}{n t_{p} w d_{33, m} d_{33, p}}\right)^{2} e_{n_{-} \text {amp }}^{2}(f)
\end{gathered}
$$

In equation (14), the equivalent magnetic noise density is represented by five physical noise sources. The first and fourth terms consist of the current noise of amplifier, the thermal noise from the bias resistance and the voltage noise of amplifier. The second and third terms present the noise sources of ME laminates. The noise floor can be estimated from Eq. (14) for ME laminates connected to a typical charge amplifier. We find that the noise contributions of the current noise of the amplifier $i_{n-a m p}$ and of the thermal noise of resistance $R_{1}$ are frequency dependent noise sources, decreasing as $1 / \mathrm{f}$ with the frequency. The piezoelectric loss noise source contribution has a $1 / \sqrt{ } \mathrm{f}$ frequency behavior. Finally, the mechanical noise and the voltage noise of amplifier have white contributions. According to the theoretical expression of equation (14), we expect the current noise of amplifier or the piezoelectric loss noise to determine the low frequency noise floor whereas, the mechanical noise or voltage noise of the amplifier may constitute the high frequency noise floor. With the significant progress in amplifier design techniques, it is possible to achieve the measurement of the ME laminates intrinsic noise limit by using low noise JFET charge amplifiers. This intrinsic noise will show a limit having a $1 / \sqrt{ } \mathrm{Hz}$ behavior at low frequencies and a white noise limit at higher frequencies. Notably, the output voltage noise is amplified by the mechanical. Considering a given shape long-type L-L push pull ME laminates, the total equivalent magnetic noise spectral density is dominated by the segment number $m$ as a function of $1 / \sqrt{ } m$ for laminates noise sources and as a function of $1 / m$ for amplifier electronics noise sources.

\section{COMPARISONS OF EXPERIMENTAL AND THEORETICAL RESULTS}

The magnetic signal sensitivity and noise floor of Metglas-PMN laminates in a L-L push pull mode connected to a low noise charge amplifier were measured at room temperature. The ME laminates were biased under an optimal DC magnetic field to achieve their maximal magnetic signal sensitivity. The bias resistance $R_{1}$ and capacitor $C_{1}$ of the charge amplifier are respectively $1 \mathrm{~T} \Omega$ and $10 \mathrm{pF}$. The gain of the second stage amplifier was equal to 50 . 
In figure 3(a), we can see that the transfer function for the ME laminates of Metgals$\mathrm{PMN}$ is about $122 \mathrm{MV} / \mathrm{T}$ with our low noise charge amplifier electronics. This means that a giant ME charge coefficient of $24.5 \mu \mathrm{C} / \mathrm{T}$ is achieved at sub-resonant frequencies for Metglas-PMN laminates under the optimal DC magnetic bias field. Please, note that low cutoff frequency observed on the different measured transfer function curves results from the second stage.

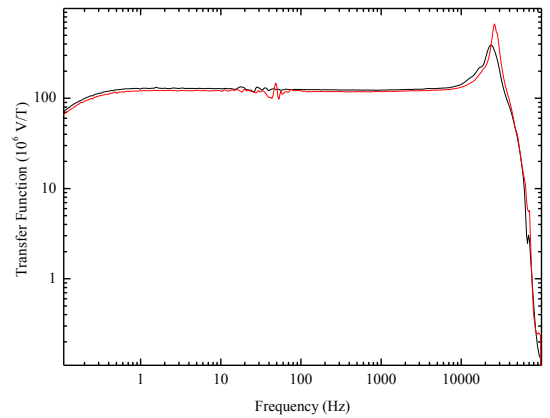

(a)

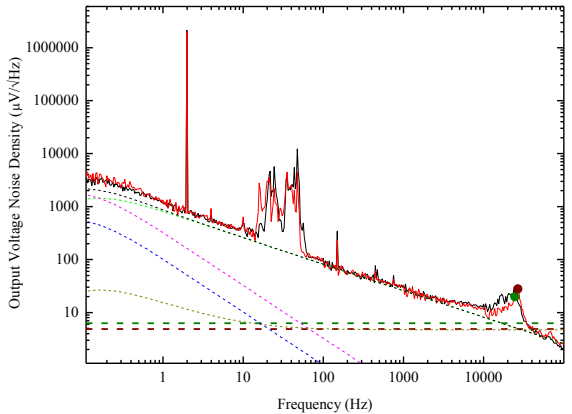

(b)

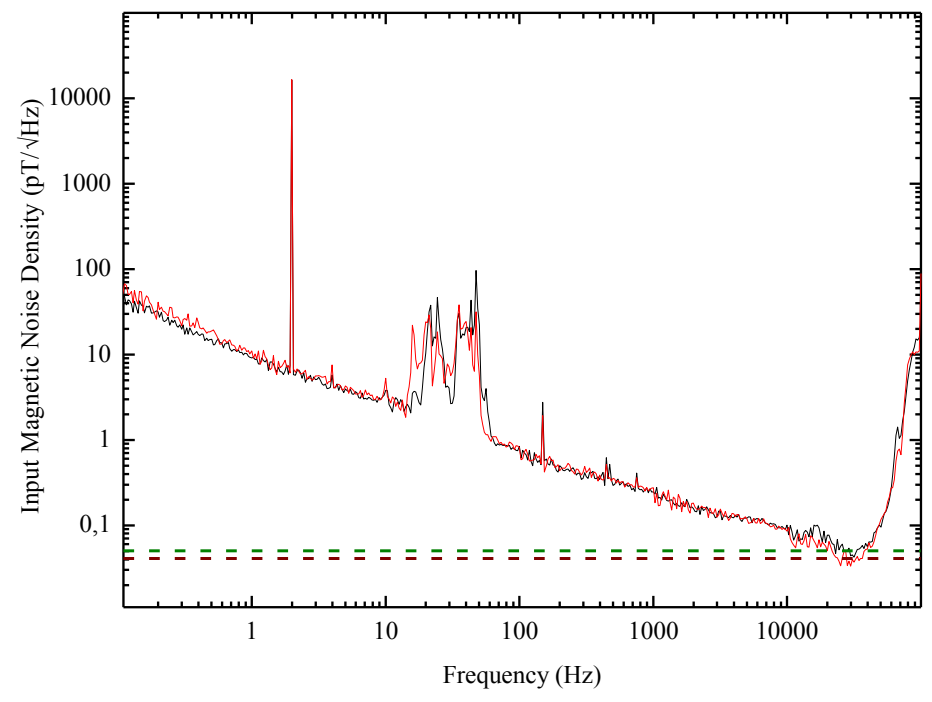

(c)

Figure 3 (a) Magnetic transfer function $T_{r}$, (b) output voltage noise spectral density $e_{n}$ and (c) equivalent input magnetic noise spectral density. The black and red curves correspond to two different laminate samples. The green, pink, yellow and blue dashed lines of figure (b) correspond respectively to the dielectric loss noise, current and voltage noise from the amplifier and the thermal noise of $R_{l}$. The dashed dark green and dark red lines represent the predicted mechanical noise floor for the corresponding black and red curves for the two samples.

The noise measurement of ME laminates with a low noise charge amplifier is a quite difficult task in the presence of various perturbations, such as magnetic, electric and vibration influences. A good magnetic shielding condition is essential in order to avoid the magnetic perturbations. In addition, a quiet measurement environment is important for minimizing the 
vibration perturbations during the measurements. The system transfer function and the output voltage noise spectral density were measured at the output terminal of the electronics using a HP 3562A dynamic signal analyzer. The ME charge coefficient $\alpha_{M E}^{Q}$ can be calculated from equation (1) and the equivalent magnetic noise floor is given by $b_{n}=\frac{e_{n}}{T_{r}}$. The two ME laminates used for our experiments have the following parameters, the values given in parentheses are for the second sample, $\alpha_{M E}^{Q}=25.5 \mu \mathrm{C} / \mathrm{T}(24.5 \mu \mathrm{C} / \mathrm{T}), C=600 \mathrm{pF}, f_{\text {res }} \frac{\omega_{\text {res }}}{2 \pi}=24.5 \mathrm{kHz}(26.7 \mathrm{kHz})$, $\tan \left(\delta_{\text {mech }}\right)=Q_{\text {mech }}^{-1}=0.256(0.07), \tan \left(\delta_{\text {piezo }}\right)=0.017$. The charge amplifier has the following parameters: a transfer function of $10^{11} \mathrm{~V} / \mathrm{C}, R_{1}=1 \mathrm{~T} \Omega, i_{n \_ \text {amp }}=0.4 \mathrm{fA} / \sqrt{\mathrm{Hz}}, e_{n_{-} \text {amp }}=1.57$ $\mathrm{nV} / \sqrt{\mathrm{Hz}}$. Figure 3(b) shows the output voltage noise spectral density $e_{n}$ measured by the HP dynamic analyzer. The measured noise floor is mainly dominated by the ME laminates intrinsic noise as expected from the theoretical equation (12). Thus, we observe the dielectric loss noise limit at low frequencies and the mechanical noise limit, amplified by the resonance, at higher frequencies. Figure 3(c) represents the equivalent input magnetic noise floor as a function of the frequency with solid lines and the mechanical noise limit in horizontal lines. Around the resonant frequency, the mechanical noise sources determine the noise floor as expected.

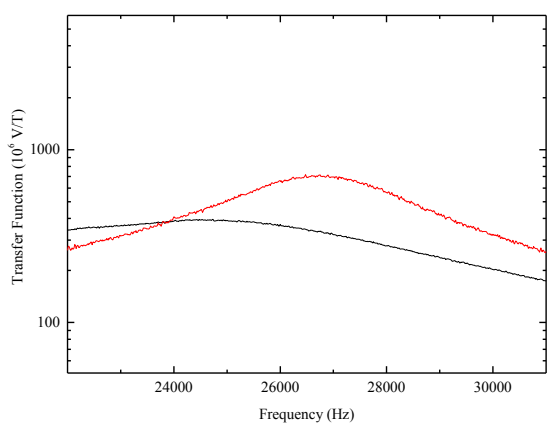

(a)

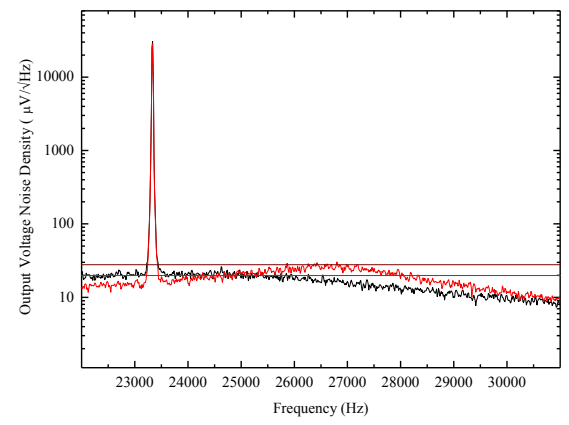

(b)

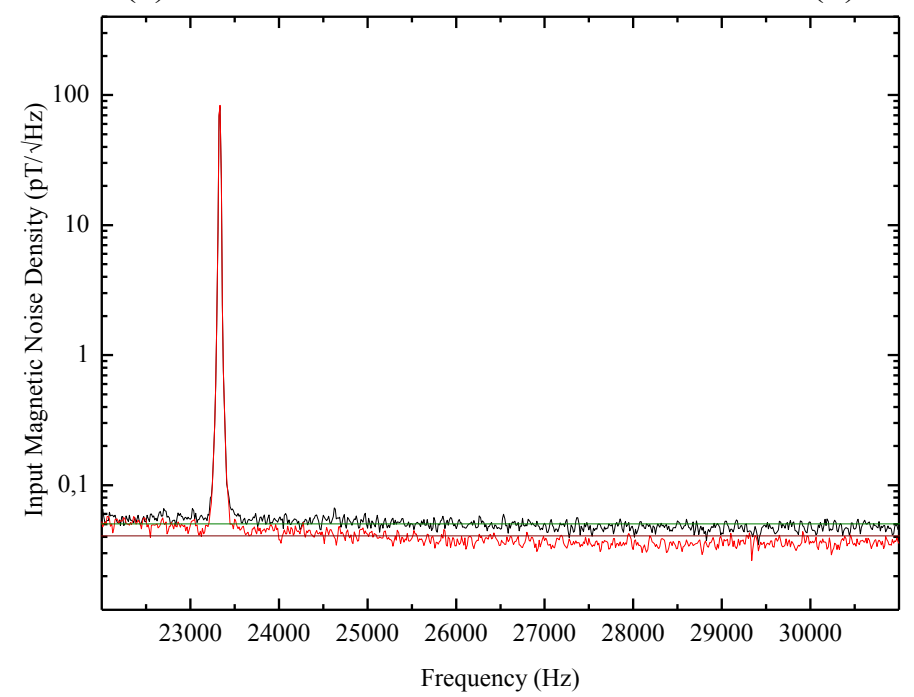

(c)

Figure 4 (a) Magnetic transfer function, (b) output voltage noise spectral density and (c) equivalent input magnetic noise spectral density of the two samples around their resonance 
frequency. The black and red solid curves correspond to the two laminate samples. The horizontal dark green and dark red lines are the predicted mechanical noise floor for the black and red curves.

For the purpose of detailing the measurement around resonant frequency, the sensitivity and noise curves around the resonant frequency were also measured as a function of the frequency. The system transfer function, the output voltage noise and the equivalent magnetic noise are shown in figure 4(a), 4(b) and 4(c). The dark red and dark green lines are the theoretical prediction noise levels referring to the mechanical noise sources.

\section{DISCUSSION AND CONCLUSION}

According to the sensitivity and noise analysis of ME laminates with an associated low noise charge amplifier we conclude that 1 ) the low frequency noise is dominated by the thermal noise of the bias resistance of the electronics, the dielectric loss noise in the ME laminates, the equivalent current noise of the amplifier or the extrinsic excess noise. Firstly, in a magnetic shielded and quiet measurement environment, magnetic and vibration perturbations could be supposed much lower than intrinsic laminates and electronics noise. Secondly, with a well designed low noise charge amplifier noise contributions from the thermal noise of bias resistance and the equivalent current noise from amplifier are lower than ME laminates noise contributions. If the above two points are achieved, the low frequency noise from ME laminates can be measured without difficulty. 2) In the white frequency bandwidth, the theoretical formula and measurements predict that both the equivalent voltage noise of amplifier and mechanical noise of laminates may determine the overall noise floor. Around the resonant frequency, mechanical noise amplified by the mechanical resonance can exceed other noise source contributions and becomes the main noise contribution. This is a significant remark in the fabrication process of ME laminates, notably for miniature ME laminates.

\section{ACKNOWLEDGMENTS}

This work was supported by the U.S. Defense Advanced Research Projects Agency (DARPA).

\section{REFERENCES}

1. C. Nan, Phy. Rev. B 50, 6082 (1994).

2. S. Dong, J. Li, and D. Viehland, J. Appl. Phys. 95, 2625 (2004).

3. M. Fiebig, J. Phys. D: Appl. Phys. 38, R 123 (2005).

4. C. Nan, M. I. Bichurin, S. Dong, D. Viehland, and G. Srinivasan, J. Appl. Phys. 103, $031101(2008)$.

5. G. Srinivasan, E. T. Rasmussen, B. J. Levin, and R. Hayes, Phys. Rev. B 65, 134402 (2002).

6. Z. Xing, J. Zhai, S. Dong, J. Li, D. Viehland, and W. G. Odendaal, Meas. Sci. Technol. 103, 033903 (2008).

7. Z. Xing, J. Zhai, J. Gao, J. Li, and D. Viehland, IEEE Electron Device Lett. 30, 445 (2009). 
8. X. Zhuang, M. Lam Chok Sing, C. Cordier, S. Saez, C. Dolabdjian, J. Das, J. Gao, J. Li, and D. Viehland, IEEE Sensors 11, 2183 (2011).

9. S. Dong, J. Zhai, J-F. Li, and D. Viehland, Appl. Phys. Lett. 89, 122903 (2006).

10. J. Das, J. Gao, Z. Xing, J. Li, and D. Viehland, Appl. Phys. Lett. 95, 092501 (2009).

11. S. K. Mandal, G. Sreenicasulu, V. M. Petrov, and G. Srinivasan, Appl. Phys. Lett. 96, 192502 (2010).

12. Y. Wang, D. Gray, D. Berry, J. Cao, M. Li, J. Li, and D. Viehland, Advanced Materials 23, 4111 (2011).

13. N. J. Kdner, Z. J. Homrighaus, T. O. Mason, and E. J. Garboczi, Thin Solid Films 496, 539 (2006).

14. F. A. Levinzon, IEEE Sensors J. 5, 1235 (2005).

15. M. Maglione, and M. A. Subramanian, Appl. Phys. Lett. 93, 032902 (2008).

16. Z. Xing, J. Li, and D. Viehland, Appl. Phys. Lett. 91, 142905 (2007).

17. T. B. Gabrielson, IEEE TRANS. ELECTRON DEV. 40, 903 (1993).

18. X. Zhuang, M. Lam Ckok Sing, C. Cordier, S. Saez, C. Dolabdjian, L. Shen, J. Li, and D. Viehland, IEEE Sensors 11, 2266 (2011).

19. X. Zhuang, M. Lam Chok Sing, S. Saez, C. Cordier, C. Dolabdjian, J. Gao, J. Li, and D. Viehland, J. Appl. Phys. 109, 124512 (2011). 